\title{
Single-Electron Charging and Coulomb Interaction in InAs Self-Assembled Quantum Dot Arrays
}

\author{
G. Medeiros-Ribeiro \\ Materials Department, University of California, Santa Barbara CA 93106 \\ F. G. Pikus \\ Department of Physics, University of California, Santa Barbara CA 93106 \\ P. M. Petroff \\ Materials Department, University of California, Santa Barbara CA 93106 \\ A. L. Efros \\ Department of Physics, University of Utah, Salt Lake City, UT 84112
}

(June 8, 2021)

\begin{abstract}
Sequential single-electron charging is observed in InAs Self-Assembled Quantum Dots using capacitance spectroscopy. In this system, the Coulomb energy is smaller than the inter-level energy spacings due to the quantum confinement and both effects can be separately identified. A theoretical model is proposed for this system and the capacitance experiments were devised in order to experimentally observe the effects of Coulomb interaction between electrons on the dots. The effects of inter- and intra-dot Coulomb interaction have been observed in the capacitance spectra. A good agreement between the proposed model and experiment is achieved.
\end{abstract}

\section{INTRODUCTION}

Quantum effects and Coulomb effects in zero dimensional systems have been extensively studied in the past years due mainly to the new and interesting phenomena these structures exhibitil. The degree of importance of each of these effects will depend on the size of the objects that form the system, since Coulomb effects should diminish linearly with the inverse of the dimensions of the system whereas quantum effects diminish quadratically. Artificially produced systems, such as those defined by electron beam lithography and other patterning techniques as well as in structures with split gate geometryl, are usually constrained by technological limitations. The ultimate size one obtains with such techniques is still too large to make quantum energies comparable to thermal energies beyond the $\mathrm{mK}$ range, and as a rule Coulomb energies prevail in these systems. Nevertheless, systems where the growth kinetics naturally produce small structures offer the possibility of decreasing even further the dimensions. As an example, islanding in highly strained heteroepitaxial coherent III-V systems 1 produces structures with dimensions were quantum effects are routinely abservable 0 , and furthermore, at elevated temperatures 8 . In this work, the InAs selfassembled quantum dots grown on GaAs 8 are investigated with capacitance spectroscopy. Previous works on this system using the capacitance technique were focused on the magneto-optical properties 9 and electronic structure 10 of the dots. The focus on the present work is on the electrostatic interactions in this system, where we analyze the effect of having a finite number of electrons in a single dot and the adjoining consequences on the capacitance spectra of having their correspondent image charges in a nearby electrode and electrons in neighboring dots. It is demonstrated that the static characteristics show the independent loading of single electrons in arrays of about $10^{9}$ dots. We successfully predicted and simulated the effects of the Coulomb interaction between electrons in the dots with the gate and electrons in other dots. The structure of this paper is as follows: in the experimental section the growth and the capacitance experiment will be described, along with the identification of the components inherent to each capacitance spectrum. The theoretical model section describes the model utilized to predict and simulate the capacitance spectra and the electrostatic effects, and the results and analysis section presents the measured data and the simulation results.

\section{EXPERIMENT}

The samples studied in this work were grown on a Varian GEN-II MBE system on semi-insulating [100] oriented 2" GaAs substrates. The conduction band diagram of a sample is shown schematically in Fig. 1. The ropeth procedure was the same as used in other works 5 . 9.10 .12 , consisting on the oxide desorption under $10^{-5}$ Torr As 4 flux and subsequent growth of a 1 micron undoped GaAs buffer layer at $1 \mathrm{ML} / \mathrm{s}$ at $620^{\circ} \mathrm{C}$. We also used 40 periods of a $2 \mathrm{~nm} \times 2 \mathrm{~nm} \mathrm{AlAs} / \mathrm{GaAs}$ short period superlattice (SPS) to trap defects and smooth the surface prior to the deposition of a 20-100 $\mathrm{nm}$ GaAs undoped layer, 20$80 \mathrm{~nm}$ thick $2 \times 10^{18} \mathrm{~cm}^{-3} \mathrm{n}$-doped back contact and a undoped GaAs tunneling barrier with thickness $d$. The 


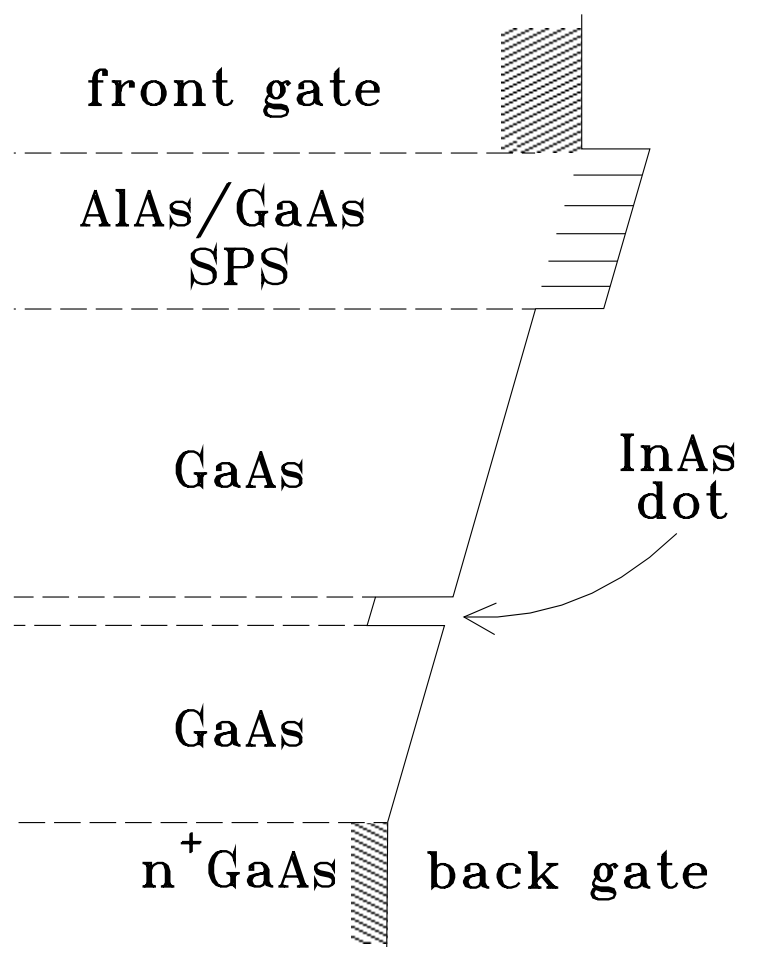

FIG. 1. Sketch of the band diagram of the quantum dot array sample in the direction perpendicular to the dot and gate planes.

growth temperature is now reduce to $530{ }^{\circ} \mathrm{C}^{12}$ and $\mathrm{InAs}$ is deposited, with the substrate fixed with the $1 \overline{1} 0$ direction aligned with the axis of the In cell. By doing that, we could produce a variation on the deposited thickness of InAs of up to 10\%, assuming an ideal point source for the effusion cell. This procedure allowed us to obtain a the dot density ranging from 0 in one side of the wafer up to $2 \cdot 10^{10} \mathrm{~cm}^{-2}$, as estimated by TEM and capacitance measurements. The growth was then resumed by depositing GaAs at $530{ }^{\circ} \mathrm{C}$ with the substrate now rotating. After $5 \mathrm{~nm}$ of GaAs thickness, the temperature was increased to $600{ }^{\circ} \mathrm{C}$ remaining at this value for the remaining part of the growth. A $25 \mathrm{~nm}$ thick GaAs was the deposited, and another AlAs/GaAs $3 \mathrm{~nm} \times 1 \mathrm{~nm}$ SPS was grown before capping the sample with a $5 \mathrm{~nm}$ thick GaAs layer.

When analyzing the experimental data, one should take into account that the potential in the dot plane $V$ is not equal to the voltage on the gate $V_{g}$. If the dots are located at a distance $d$ from the back gate and the distance between the front and back gates is $t$ than

$$
\frac{V_{g}}{V}=L \equiv \frac{t}{d}
$$

We call the ratio $L$ a lever-arm coefficient, it varies from 6 to 7 in different samples. We kept values of $L$ similar for all samples with different $d$ by varying the thickness of the top AlAs/GaAs SPS.

Samples with tunneling barriers thinner than $20 \mathrm{~nm}$

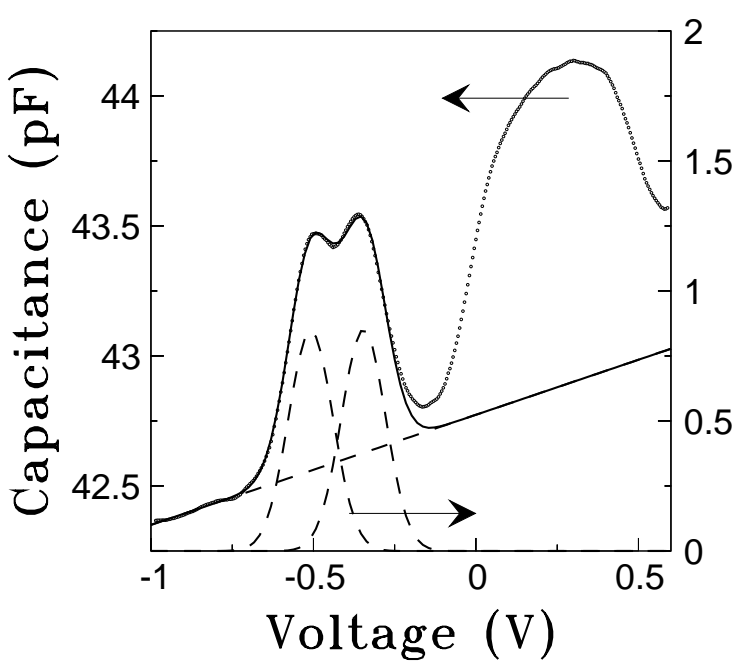

FIG. 2. Dot capacitance vs gate voltage for the samples with the distance $d=450 \AA$ between dot and gate planes. Circles show the experimental data, solid line gives the fit by the sum of the linear background and two Gaussian peaks (shown by dashed lines).

were not used since the strain field produced by the dots extends into the substrate for approximately this amount, as qualitatively inferred by changes in contrast in cross-section TEM micrographs. The thickness of the back-contact had also to be increased so that the strain field would not deplete the regions beneath the dots and therefore producing effectively different $d$ values.

Metallic disks were evaporated after contacting the back contact layer using standard procedures for making ohmic contacts in $\mathrm{n}$ doped GaAs. The photolithographically defined disks consisted of a thin layer of $\mathrm{Cr}(5-10$ $\mathrm{nm}$ ) followed by a $200 \mathrm{~nm}$ thick Au layer, with diameter from $150 \mu \mathrm{m}$ to $350 \mu \mathrm{m}$. The diodes were bonded and their capacitance was measured at $4.2 \mathrm{~K}$ in a liquid $\mathrm{He}$ immersion cryostat. To obtain the capacitance voltage characteristics of the devices, we used a lock-in amplifier at frequencies varying from $40 \mathrm{~Hz}$ to $30 \mathrm{kHz}$. The ac amplitude was kept smaller than $1 \mathrm{meV}$, after conversion into energy by the lever arm coefficient. Depending on the thickness of the tunneling barrier $d$ we used higher or lower frequencies, choosing the appropriate value to have the loading and unloading of the dots within one period of the ac frequency, i.e., $f<2 \pi R C$, where $R$ is the tunneling resistance and $C$ is the capacitance determined by the area of each dot and the distance $d$ from the back contact.

In Fig. 2 we show the measured capacitance-voltage characteristics of the sample A with $d=450 \AA$. One can see the two peaks, corresponding to the loading of the ground and first excited states of the dots. The first peak is split into two; this splitting results from the Coulomb interaction of the electron in the same dot. In this paper we analyze only this split peak, related to the ground state in the dots. 
The following problem complicates a direct comparison of the experimental results with theoretical capacitances: the experimentally measured capacitance $C_{\exp }$ contains a large background capacitance $C_{\mathrm{b}}$, to which the capacitance of the dots $C_{\text {dot }}$ is a small correction:

$$
C_{\text {exp }}=C_{\mathrm{b}}+C_{\text {dot }} .
$$

We resolve this problem by assuming a linear background capacitance $C_{\mathrm{b}}$ and extrapolating it using the low-voltage part of the CV curve. For the sample A we have also measured the capacitance at high frequency of the ac voltage. In this case the electrons do not have the time to tunnel to and from the dots during one period of the ac voltage, and the dots essentially do not contribute to the capacitance, whereas the background capacitance has no intrinsic slow processes, and does not depend on the frequency in a wide frequency range. Thus measured background capacitance shows a linear voltage dependence for the voltages where at low frequencies the dot capacitance is observed. We have also used a background capacitance in the form of the capacitance of a depletion layer, which is formed in the doped back contact:

$$
C_{\mathrm{b}}^{\prime}=\frac{S V_{d}}{\left(V-V_{0}\right) d}\left[\sqrt{1+\frac{V-V_{0}}{2 \pi V_{d}}}-1\right],
$$

where $V_{d}=e^{2} N_{d} / \kappa, N_{d}$ is the density of the positive charge in the depletion layer, $\kappa$ is the dielectric constant, $V_{0}$ is the voltage of flat bands, and $S$ is the area of the sample. In the range of $V$ where the capacitance peak is observed (and for smaller voltages), this function can be considered as a linear one. All three ways of extracting the background capacitance lead to practically the same results.

After the background is subtracted, the capacitance correction due to quantum dots $C_{\text {dot }}$ can be very well described by a sum of two Gaussian peaks with centers at voltages $V_{1}$ and $V_{2}$ and widths $\sigma_{1}$ and $\sigma_{2}$, respectively:

$$
C_{\text {dot }}=\frac{Q_{0}}{\sqrt{2 \pi}}\left[\frac{1}{\sigma_{1}} \mathrm{e}^{-\frac{\left(V-V_{1}\right)^{2}}{2 \sigma_{1}^{2}}}+\frac{1}{\sigma_{2}} \mathrm{e}^{-\frac{\left(V-V_{2}\right)^{2}}{2 \sigma_{2}^{2}}}\right] .
$$

This fit reflects the fact that the areas of both peaks $Q_{0}$ should be equal, since they give the total charge of all dots with one electron per dot. The fitting was done by weighted explicit orthogonal distance regression using the software package ODRPACK16. The resulting decomposition of the experimental curve into linear background and two Gaussian peaks is shown in Fig. 2.

\section{THEORETICAL MODEL}

We now present a theoretical description of the quantum dot array in the framework of the following Hamiltonian:

$$
\begin{aligned}
H= & \sum_{i} n_{i} \varphi_{i}+\frac{e^{2}}{\kappa} \sum_{i} \frac{n_{i}\left(n_{i}-1\right)}{2 C_{i}}-\frac{e^{2}}{\kappa} \sum_{i} \frac{n_{i}^{2}}{4 d}+ \\
& \frac{e}{\kappa} \sum_{i<j} n_{i} n_{j} V\left(r_{i j}\right)-e V \sum_{i} n_{i} .
\end{aligned}
$$

Here the indices $i, j$ number the quantum dots. $n_{i}$ is the occupation number of the $\operatorname{dot} i$; in this paper we only consider the ground state on the dot, so $n_{i}$ can be 0,1 , and 2 . The first term represents the non-Coulomb disorder due to fluctuations of size quantization energies $\varphi_{i}$ in the dots. We assume here a Gaussian distribution for $\varphi$ :

$$
\omega(\varphi)=\frac{1}{\sqrt{2 \pi} \sigma_{\varphi}} \mathrm{e}^{-\frac{\varphi^{2}}{2 \sigma_{\varphi}^{2}}}
$$

The width $\sigma_{\varphi}$ describes the magnitude of disorder. It is a fitting parameter in our model. The second term in the Hamiltonian Eq. (3) is the charging energy of the dot (this term is responsible for Coulomb blockade-like effects). $C_{i}$ is the capacitance of the dot, defined so that

$$
W=\frac{e^{2}}{\kappa C}
$$

is the energy of Coulomb interaction of two electrons in the dot 13 . The product $n_{i}\left(n_{i}-1\right)$ takes into account that electron does not interact with itself. In our model, we take $C_{i}=C$ to be the same for all dots, and use it as another fitting parameter 14 . The third term in the Hamiltonian is the attraction energy between the charge of the dot and its image in the metallic electrode at a distance $d$ from the dot plane. We have considered only the attraction to the closest metallic electrode, which is the back gate in our samples. The front gate is much further away from the dots, and has no significant effect on the electrostatics near the dots. The last term introduces the effect of the gate bias $V$ (scaled by the lever-arm ratio Eq. (11)).

The fourth term of the Hamiltonian Eq. (3) takes into account the interaction between the charges on different dots. The interaction potential $V(r)$ includes the screening of the Coulomb interaction by the gate and has the form:

$$
V(r)=\frac{e}{\kappa}\left[\frac{1}{r}-\frac{1}{\sqrt{r^{2}+4 d^{2}}}\right] .
$$

Here we again neglect the effects of the second, remote gate. We should also mention that we have assumed the point charge Coulomb interaction of the dots with the gate and with each other The latter assumption is well justified when the dot size is much smaller than the distance between the dots, which is the case in all of our samples. For the dot-gate interaction, a trivial calculation shows that the interaction of an electron wave function in a disk of size $a$ with the gate at a distance $d$ 
differs negligibly from that of a point charge when $d \geq a$. This condition also holds for all of samples for which we present the results here.

The thermodynamic properties of the many-electron system with the Hamiltonian Eq. (3) in the regime when the Coulomb interactions are essential are rather complex, and a comprehensive solution is possible only by means of numerical modeling. The modeling consists of the following steps:

1) Generation of dot positions and energies. The given number of $\operatorname{dots} N$ are placed inside a square of the size $L$. The dot density $N_{\text {dot }}=N / L^{2}$ for our samples is about $10^{10} \mathrm{~cm}^{-2}$, and we can simulate systems of $N=2500-10000$ dots. The positions of the dots are random, with one restriction: no two dots can be closer to each other than the minimum dot separation $r_{\min }$. The results weakly depend on $r_{\min }$; we can deduce its approximate value $r_{\min }=250 \AA$ from the TEM data for our samples. Also at this step we generate Gaussian random energies $\varphi_{i}$.

2) Monte-Carlo step. On this step we use the Metropolistype Monte-Carlo algorithm to calculate an average density of electrons on the dots $n=\left\langle n_{i}\right\rangle$ for a given gate voltage $V_{g}$ and temperature $T$. In this paper we have not studied the temperature dependence of the capacitance, and the temperature was maintained at $T=4.2 \mathrm{~K}$. We employ the parallel version of Metropolis algorithm for Coulomb system 15 . The gate voltage is being slowly varied across the desired interval, so that the system has enough time to reach equilibrium for each value of $V_{g}$.

3) Calculations of capacitance. In order to find the capacitance $C_{\mathrm{dot}}$ of the dots as a function of gate voltage, we compute the derivative $\frac{e d n}{d V}$. It is easy to show that the correction to the capacitance per unit area can be calculated from the equation

$$
C_{\mathrm{dot}}(V)=\frac{e d n}{d V} \frac{d}{t}\left(1-\frac{d}{t}\right) .
$$

The voltage $V$ is connected to the gate voltage $V_{g}$ by Eq. (1). This capacitance has a lot of "noise" unless the temperature is very high or the number of $\operatorname{dots} N$ is large, and we get a smooth curve by convoluting it with a Gaussian filter with small width. The results of these computations are then compared with the experiment.

The computations were done on the Intel Paragon using 128 nodes. It takes about 6 hours to compute one C-V curve.

\section{RESULTS AND ANALYSIS}

We begin by comparing the experimental CV-curves with the results of our modeling (Fig. 3). First of all, we subtract the background capacitance from the experimental data as described above. Since the areas of the real and simulated devices are different, so the two capacitances cannot be directly compared. We therefore

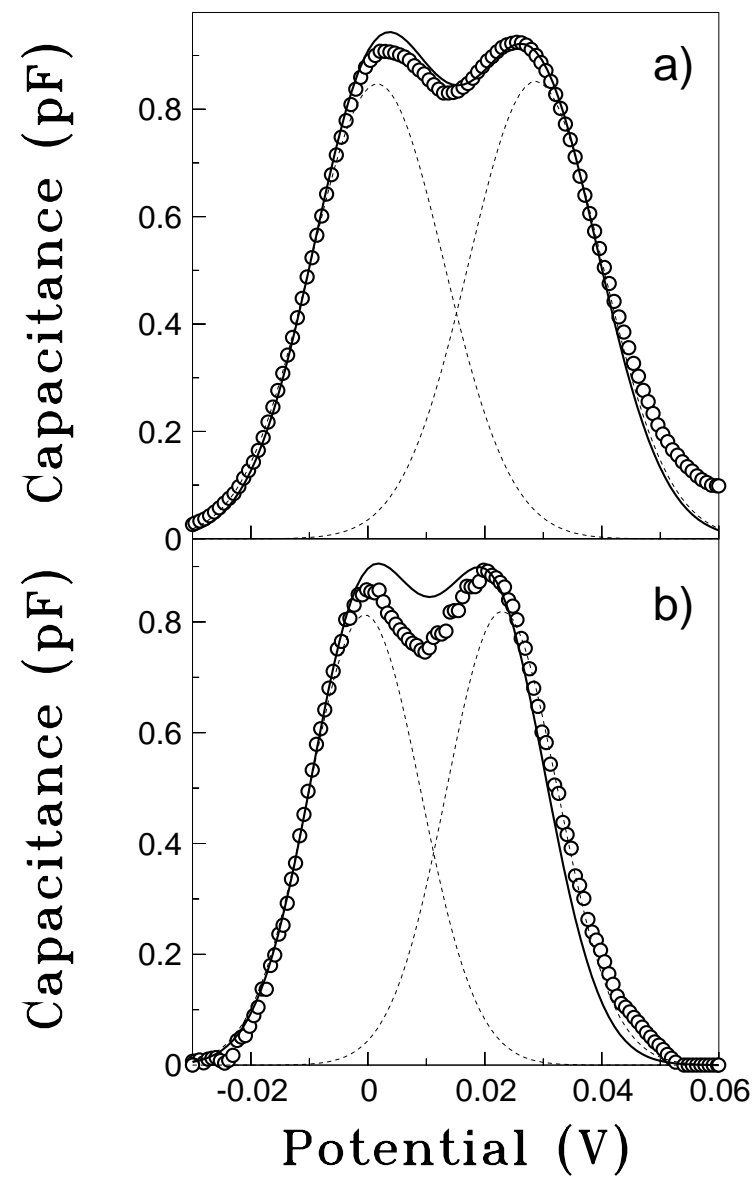

FIG. 3. Dot capacitance vs potential in the dot plane for two samples with different distances $d$ between dot and gate planes: a) - A, $d=450 \AA$, b) - B, $d=200 \AA$. Circles show the experimental data with background capacitance subtracted, solid lines give the results of our modeling with non-Coulomb disorder $\sigma=8.5 \mathrm{meV}$ and dot charging energy $W=e^{2} / \kappa C=23 \mathrm{meV}$.

normalize the calculated capacitance so that the areas under the curve, which give the total charge accumulated on the dots at large voltages, are the same. We have checked that the area of the experimental capacitance peak corresponds to two electrons per dot for our sample sizes and dot density $N_{\text {dot }} \approx 10^{10} \mathrm{~cm}^{-2}$, which is in good agreement with the TEM measurements of $N_{\mathrm{dot}}$. We also convert the gate voltages to the potentials in the dot plane using the lever-arm coefficient Eq. (1).

The Fig. (3) shows the measured (circles) and computed (lines) dot capacitances for two samples with different dot to gate distances $d=450 \AA$ and $d=200 \AA$. The parameters of the modeling were chosen to get a best fit for the sample A with $d=450 \AA$ (Fig. (3a): dot charging energy $W=e^{2} / \kappa C=23 \mathrm{meV}$ and non-Coulomb disorder magnitude $\sigma_{\varphi}=8.5 \mathrm{meV}$. We then computed the capacitance for the second sample, B with $d=200 \AA$, using the same parameters. One can see from Fig. (3) b that the theoretical and experimental capacitances agree 
quite well. Since both samples were grown under similar conditions and differ only in the thickness of the tunneling layers, it is reasonable to assume that the sizes of the dots and their fluctuations should be the same for both samples. Therefore, the ability of our model to reproduce the effect of the change of $d$ on the capacitance, which is quite substantial, without any additional parameters should be considered as an evidence in favor of the model.

We now turn to discussing the effects of the Coulomb interaction on the dot capacitance. First of all, the Coulomb interaction of two electrons in one dot is responsible for the structure of the capacitance peaks. In the absence of disorder the capacitance would exhibit two delta-function peaks separated by the dot charging energy. These peaks are located at those values of gate voltage $V_{g 1}$ and $V_{g 2}$ at which first and second electrons, respectively, enter the dot. The corresponding potentials in the dot plane are $V_{1}=V_{g 1} / L$ and $V_{2}=V_{g 2} / L$. The disorder broadens the peaks, however, they are still observable in our samples. The charging energy depends first and foremost on the dot shape. It has, however, a correction, which comes from the interaction of the charges in the dot with their electrostatic images in the metallic gate. It is easy to show that this correction reduces the voltage between the capacitance peaks $\delta V=V_{2}-V_{1}$ by

$$
\delta V_{\text {gate }}=-\frac{e}{2 \kappa d} .
$$

This correction leads to the observed dependence of the peak splitting on the distance $d$ between the dots and the gate.

This picture is further complicated by the interaction of electrons on different dots. This interaction gives rise to two effects. First, in order to load an electron into a dot, one has to overcome the repulsion of all charges already on the dots. This effect shifts the position of capacitance peaks to higher voltages. The second peak is shifted much more than the first one, since the shift is proportional to the charge density already on the dots. Therefore, the dot-dot interaction increases the splitting between capacitance peaks $\delta V$. This correction to $\delta V$, which we call $\delta V_{\text {int }}$, is also $d$-dependent: the smaller is $d$ the weaker is the screened Coulomb interaction and the smaller is the correction. One can see that the $d$ dependence of $\delta V_{\text {int }}$ and $\delta V_{\text {gate }}$ are the same - the peak splitting increases at large $d$ due to both corrections. A very rough estimate of $\delta V_{\text {int }}$ can be obtained by calculating the average potential created by a random set of singly charged dots (with minimal distance between nearest dots $\left.r_{\min }\right)$ :

$$
\begin{aligned}
\delta V_{\mathrm{int}}= & N_{\mathrm{dot}} \int V(r) d^{2} r= \\
& \frac{2 \pi e}{\kappa} N_{\operatorname{dot}}\left(\sqrt{4 d^{2}+r_{\min }^{2}}-r_{\min }\right) .
\end{aligned}
$$

The second effect of the interdot interaction also has to do with the potential created by the charges on the

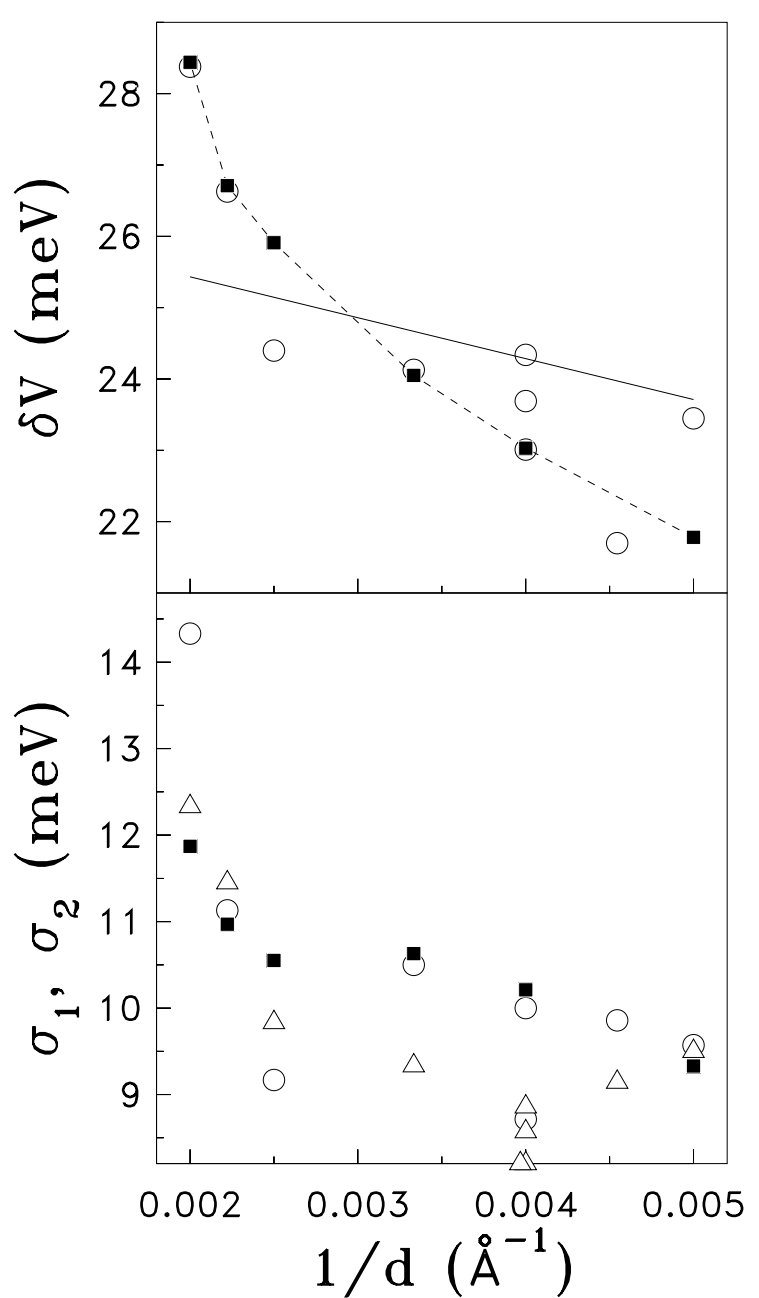

FIG. 4. Capacitance peaks splitting $\delta V$ (a) and peak widths $\sigma_{1}$ and $\sigma_{2}$ (b) vs the reciprocal dot-gate distance $1 / d$. The experimental values of $\delta V$ and $\sigma_{1}$ are shown by circles, those of $\sigma_{2}$ - by triangles. The results of the modeling are shown by solid squares. The modeling gives very close values of $\sigma_{1}$ and $\sigma_{2}$, so only $\sigma_{1}$ are shown. The dashed line is a guide for the eye. The solid line shows the slope of the correction $\delta V_{\text {gate }}$, Eq. (1).

dots. Since the dots are placed randomly in the plane, the potential created by them is also random. For each dot, this potential is added to the ground state energy, resulting in effectively increasing the total disorder in the system. This additional, "Coulomb disorder", is also $d$ dependent. Its order-of-magnitude estimate is provided by the dispersion of the screened Coulomb potential of randomly placed dots:

$$
\begin{aligned}
\delta \sigma_{\text {int }}^{2}= & N_{\text {dot }} \int V(r)^{2} d^{2} r= \\
& \frac{2 \pi e^{2}}{\kappa^{2}} N_{\text {dot }} \ln \left\{\frac{\left[r_{\min }+\sqrt{4 d^{2}+r_{\min }^{2}}\right]^{2}}{4 r_{\min } \sqrt{4 d^{2}+r_{\min }^{2}}}\right\} .
\end{aligned}
$$

This "Coulomb disorder" will add to the disorder caused 
by fluctuations of the size quantization energies in the dots, resulting in wider capacitance peaks.

One can easily see the above interaction effects in the modeling. For example, the theoretical curve in Fig. 3 a is obtained for charging energy $W=23 \mathrm{meV}$ and disorder magnitude $\sigma=8.5 \mathrm{meV}$, while the distance between the peaks is $\delta V \approx 24.5 \mathrm{meV}$ and peak widths are $\sigma_{1} \approx \sigma_{2} \approx 9.5 \mathrm{meV}$. However, experimentally all of the effects of the Coulomb interaction are best studied using their sensitivity to the screening by the metallic gate. All of these effects are qualitatively evident already in the Fig. 3, where one can see that for the sample A with larger $d=450 \AA$ the peaks are wider and further apart than for the sample B with smaller $d=200 \AA$.

For a quantitative analysis, in Fig. A we compare the measured and computed values of peak splitting $\delta V$ and widths $\sigma_{1}, \sigma_{2}$ for a set of samples with different dot-gate distances $d$. Again, the $\operatorname{dot}$ capacitance $C$ and the nonCoulomb disorder $\sigma$ were chosen from best fit for one sample with $d=450 \AA$, and then the results were computed for other values of $d$ without any additional adjustable parameters. One can clearly see that the peak splitting increases with $d$, and that this increase is faster than the correction $\delta V_{\text {gate }}$ (Eq. (7)) alone would give. This shows that the dot-dot Coulomb interaction, which is responsible for the second correction $\delta V_{\text {int }}$, is very important in the arrays of self-assembled quantum dots. The modeling includes both of those corrections, and it provides very good description of the experimental data. The peak widths also increase with $d$ as a result of the Coulomb interaction (see Fig. đ4b), again with modeling providing reasonably good description of the experiment.

\section{CONCLUSIONS}

We have presented a capacitance spectroscopy study of InAs self-assembled quantum dots grown on GaAs and compared the experimental results with the computer simulations. By doing such a comparison for a series of devices with different distances between the dot and gate planes, we have shown the effect of the Coulomb interactions, both inside one dot and between the dots, on the capacitance spectra.

We have found out that the Coulomb interaction provides two capacitance peaks which correspond to sequential loading of two electrons in one dot. The distance between the peaks and the width of the peaks contain an information about interactions inside one dot and between the dots. In our experiments the interdot interaction was weaker than the external (non-Coulomb) disorder. A new, interesting physics could be observed in an ordered dot array with weak disorder: then the interaction controls the distribution of electrons on the dots. This may change the sign of the derivative $\frac{e d n}{d V}$ as was predicted by Efros 17 and was observed by Eisenstein, Pfeffer, and West 18 in clean $2 D$ systems.

\section{ACKNOWLEDGMENTS}

We are grateful to W. Kohn for helpful discussions. This work was supported by the Center for Quantized Electronic Structures (QUEST) of UCSB, Grant DMR 91-2007, and by San Diego Supercomputer Center. F. G. P. acknowledges support by the NSF Grant DMR 9308011 and by the Quantum Institute of UCSB. A. L. E. acknowledges support by the Subcontract KK3017 of QUEST of UCSB. G. M. R. would like to acknowledge the financial support from $\mathrm{CNPq}$, Brazilian agency.

${ }^{1}$ T. Chakraborty, Comm. Cond. Mat. Phys. 16, 35 (1992).

${ }^{2}$ R. C. Ashoori, H. L. Stoermer, J. S. Weiner, C. N. Pfeiffer, K. W. Baldwin, and K. W. West, Phys. Rev. Lett. 71, 613 (1993).

${ }^{3}$ Reviewed in Z. Phys. B 85 (1991).

${ }^{4}$ S. Guha, A. Maduhkar, and K. C. Rajkumar, Appl. Phys. Lett. 57, 2110 (1990).

${ }^{5}$ D. Leonard, M. Krishnamurthy, C. M. Reaves, S. P. Denbaars, and P. M . Petroff, Appl. Phys. Lett. 63, 3203 (1993).

${ }^{6}$ J. M. Moison, F. Houzay, F. Barthe, L. Leprince, E. André, and O. Vatel, Appl. Phys. Lett. 64, 196 (1994).

${ }^{7}$ S. Fafard et al., submited to Phys. Rev. B.

${ }^{8}$ G. Medeiros-Ribeiro, J.M Garcia and P. M. Petroff, unpublished.

${ }^{9}$ H. Drexler, D. Leonard, W. Hansen, J. P. Kotthaus and P. M. Petroff, Phys. Rev. Lett. 73, 2252 (1994).

${ }^{10}$ G. Medeiros-Ribeiro, D. Leonard and P. M. Petroff, Appl. Phys. Lett. 66, 1767 (1995).

${ }^{11}$ S. Tiwari, F. Rana, H. Hanafi, A. Hartstein, E. Crabbé and K. Chan, Appl. Phys. Lett. 68, 1377 (1996)

${ }^{12}$ D. Leonard, K. Pond and P. M. Petroff, Phys. Rev. B 50, 11687 (1994).

13 The capacitance $C_{i}$, in fact, depends on the number of electrons in the dot, and approaches the conventional geometrical capacitance when this number is large enough. However, in this paper we only consider $n_{i} \leq 2$, so only the capacitance for adding the second electron is relevant.

${ }^{14}$ The reason for neglecting the fluctuations of the dot capacitance is as follows: the fluctuations of $C_{i}$ would have broadened the capacitance peak for the second electron, while the first peak would remain unchanged since one electron in a dot has no Coulomb energy. The experiment, however, shows that the widths of first and second peaks are very close. This implies that the fluctuations of dot capacitance are negligible.

${ }^{15}$ F. G. Pikus, News of Utah Supercomputing Institute, 5, 1 (1994).

${ }^{16}$ P. T. Boggs et. al, NIST preprint NISTIR 92-4834.

${ }^{17}$ A. L. Efros, Solid State Commun. 65, 1281 (1988); ibid 67, 1019 (1988). 
18 J. P. Eisenstein, L. N. Pfeiffer, and K. West, Phys. Rev. Lett., 68, 674 (1992). 